\title{
Continued high use of PPIs despite C. difficile risk
}

$\mathrm{P}$ roton-pump inhibitor prescriptions continue to soar despite evidence dating back to 2004 implicating the drugs in Clostridium difficile infections and subsequent measures to put a brake on prescribing. Experts on both sides of the debate agree the popular stomach-acid suppressants are still being overprescribed despite a Health Canada advisory issued in February 2012. They also agree that the high rate of seniors on proton-pump inhibitors (PPIs) raises worries about $C$. difficile outbreaks in hospitals and nursing homes.

PPIs such as esomeprazole and lansoprazole, were introduced in the early 1990s and soon displaced histamine-2 receptor antagonists as the most-used drugs for gastroesophageal reflux. The Canadian Institute for Health Information states that PPI use by seniors covered by public drug plans increased from $13.1 \%$ in $2001 / 02$ to $21.1 \%$ in 2007/08; use reached $27 \%$ by 2012 .

PPIs are invaluable for acid reflux, which can damage the esophagus and cause gastrointestinal bleeding, says Dr. Paul Moayyedi, director of the Division of Gastroenterology at McMaster University in Hamilton, Ont., but "there is no doubt they are overprescribed" for other problems for which they are less efficacious.

Concerns came to the fore in 2004, when a CMAJ article (171:33-8) showed a strong correlation between the drugs and $C$. difficile risk. About a year later, the Canadian Association of Gastroenterology issued a position statement asking members to limit prescriptions to evidence-based indications because of the $C$. difficile link.

The issue was taken on by Member of Parliament James Lunney (Conservative - Nanaimo-Alberni), who says he began to press Health Canada to take action in 2005.

That didn't happen until four years later. According to an email from Health Canada, it conducted an indepth review because of emerging evidence in 2009, which indicated that

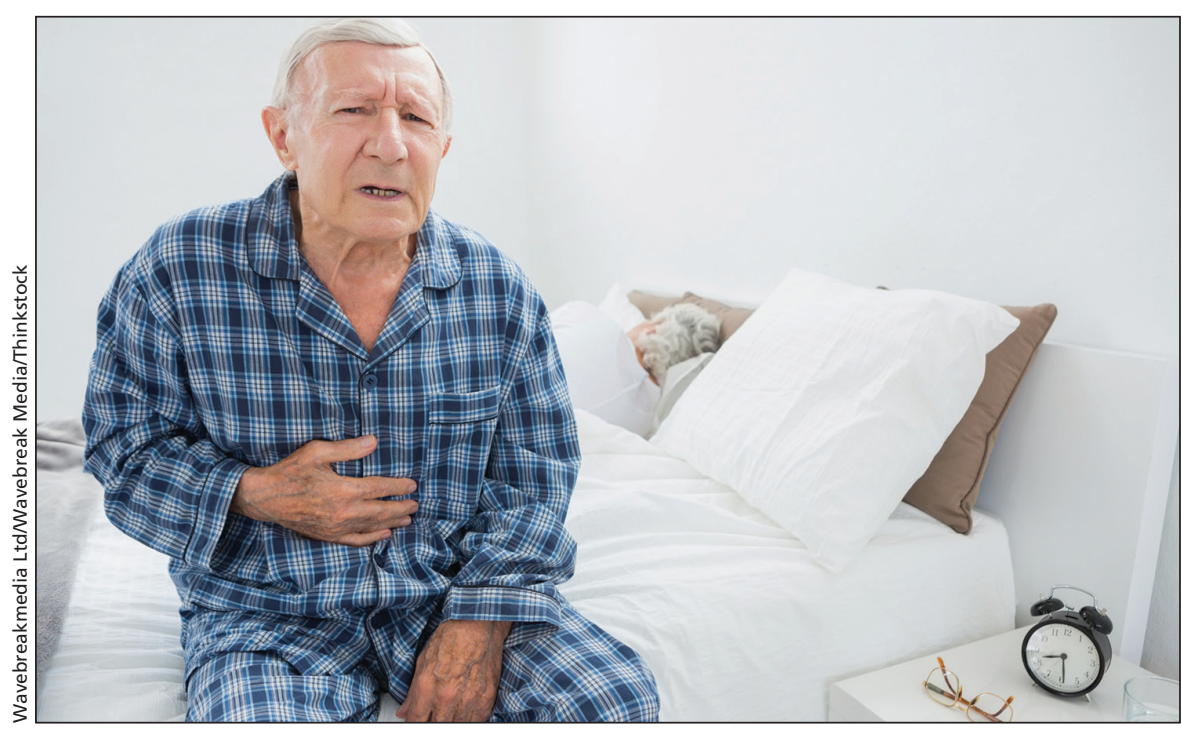

Health Canada issued a warning eight years after research showed a link between protonpump inhibitors and C. difficile.

"findings on this topic remain inconclusive." Nevertheless, in May 2010, Health Canada asked PPI manufacturers to add this statement to their product monographs: "Treatment with proton pump inhibitors may lead to slightly increased risk of gastrointestinal infections such as Salmonella and Campylobacter and possibly $C$. difficile." Health Canada also asked the Drug Safety and Effectiveness Network (DSEN) to study whether PPIs raise the risk of $C$. difficile infection.

Executive Director Dr. Robert Peterson says DSEN researchers believed that any research they could do from health data would find no more than what was already evident in the literature. DSEN's April 2012 report to Health Canada concludes that previous studies show that PPIs are "quite strongly" associated with C. difficile risk, which increases with the dose of PPI. "I can't think of another query that resulted in an outcome such as this," says Peterson.

Three months earlier, on Feb. 8, 2012, the US Food and Drug Administration had issued an advisory concerning the $C$. difficile risk from PPIs; Health Canada followed suit on Feb. 16.

Moayyedi, who conducted a system- atic review of PPIs (Nat Rev Gastroenterol Hepatol 2012;9:132-9), says "there is definitely an association" between PPIs and $C$. difficile infections. "The big question is whether that association is causal. I don't pretend to know the answer." Since C. difficile is rare in healthy people in the community, Moayyedi continues to prescribe PPIs because "there are people who need them because they improve their quality of life.'

Jason Tetro, a research microbiologist who has written articles on the issue, says, "When faced with a decision in terms of medication, a doctor needs to have something to prescribe that has been shown through Cochrane reviews and clinical trials."

However, all experts agree that high use of PPIs in hospitals and nursing homes raises the risk of serious $C$. difficile outbreaks. "In hospitals, we should take care of prescribing these drugs to the right person," says Moayyedi. Tetro adds, "We should be looking at PPI stewardship ... They should not be considered the 'go-to' treatment but the last resort." - Carolyn Brown, Ottawa, Ont. 